Claremont Colleges

Scholarship@ Claremont

All HMC Faculty Publications and Research

HMC Faculty Scholarship

$1-1-2006$

\title{
Communicating Applied Mathematics: Four Examples
}

Daniel E. Finkel

North Carolina State University at Raleigh

Christopher Kuster

Carroll University

Matthew Lasater

North Carolina State University at Raleigh

Rachel Levy

Harvey Mudd College

Jill P. Reese

See next page for additional authors

\section{Recommended Citation}

Finkel, Daniel E., Christopher Kuster, Matthew Lasater, Rachel Levy, Jill P. Reese, and Ilse C. F. Ipsen. "Communicating Applied Mathematics: Four Examples." SIAM Review 48.2 (2006): 359-389, DOI: 10.1137/S0036144504443523

This Article is brought to you for free and open access by the HMC Faculty Scholarship at Scholarship @ Claremont. It has been accepted for inclusion in All HMC Faculty Publications and Research by an authorized administrator of Scholarship @ Claremont. For more information, please contact scholarship@cuc.claremont.edu. 
Authors

Daniel E. Finkel, Christopher Kuster, Matthew Lasater, Rachel Levy, Jill P. Reese, and Ilse C. F. Ipsen 


\title{
Communicating Applied Mathematics: Four Examples*
}

\author{
Daniel E. Finkel ${ }^{\dagger}$ \\ Christopher Kuster ${ }^{\dagger}$ \\ Matthew Lasater ${ }^{\dagger}$ \\ Rachel Levy ${ }^{\dagger}$ \\ Jill P. Reese ${ }^{\dagger}$ \\ Ilse C.F. Ipsen ${ }^{\dagger}$
}

\begin{abstract}
Communicating Applied Mathematics is a writing- and speaking-intensive graduate course at North Carolina State University. The purpose of this article is to provide a brief description of the course objectives and the assignments. Parts A-D of of this article represent the class projects and illustrate the outcome of the course:

- The Evolution of an Optimization Test Problem: From Motivation to Implementation, by Daniel E. Finkel and Jill P. Reese

- Finding the Volume of a Powder from a Single Surface Height Measurement, by Christopher Kuster

- Finding Oscillations in Resonant Tunneling Diodes, by Matthew Lasater

- A Shocking Discovery: Nonclassical Waves in Thin Liquid Films, by Rachel Levy
\end{abstract}

Key words. education, mathematical writing, presentations, audience, peer critique

AMS subject classifications. 97C90, 97D30, 97D40

DOI. $10.1137 / \mathrm{S} 0036144504443523$

I. Overview. Communicating Applied Mathematics (MA798I) is a graduate course that I (I.C.F. Ipsen) teach in the Mathematics Department at North Carolina State University. It is a writing- and speaking-intensive three-credit course for Ph.D. students in applied mathematics who have passed the qualifying exams. The class web page, http://www.math.ncsu.edu/SIAM_Review_paper/ma798I, contains links to the homework assignments, as well as a list of resources on writing and presentations.

The general goal of the course is to improve the students' writing and speaking in the area of applied mathematics. In particular, the students learn to present their ideas in a linear, coherent sequence. They also learn to identify their audience and write and speak at a level appropriate for this audience. Two important ingredients of the course are repeated revisions and peer critique.

* Received by the editors April 30, 2004; accepted for publication (in revised form) November 11, 2005; published electronically May 2, 2006. The work of the first and fifth authors was supported by the Army Research Office through grant DAAD19-02-1-0391, the Department of Education through a GAANN Computational Science Fellowship, and the National Science Foundation through grants DMS-0209695, DMS-0112542, and DMS-0070641. The work of the third author was supported by the Army Research Office under a MURI (Multidisciplinary University Research Institute) contract. http://www.siam.org/journals/sirev/48-2/44352.html

${ }^{\dagger}$ Department of Mathematics, North Carolina State University, P.O. Box 8205, Raleigh, NC 276958205 (dfinkel@ll.mit.edu, cmkuster@ncsu.edu, mslasater@ll.mit.edu, rlevy@math.duke.edu, jpreese@ ncsu.edu, ipsen@ncsu.edu, http://www4.ncsu.edu/ ipsen/). 
We use two textbooks: Handbook of Writing for the Mathematical Sciences, by Nick Higham [1], and A Primer of Mathematical Writing, by Steven Krantz [2]. Class meetings consist of writing exercises (section 2), student presentations (section 3), discussions of the class project, and peer critiques (i.e., the students critique each other's presentations or writing). There are one or two homeworks per week plus a class project. Homeworks (section 4) are devoted to preparing presentations, explaining one's work to nonmathematicians, understanding and analyzing papers, reviewing papers, preparing a CV, and working on the class project. The class project (section $5)$ consists of writing a paper for a general mathematical audience.

In 2004 the students in this course were Dan Finkel, Chris Kuster, Matthew Lasater, Rachel Levy, and Jill Reese. The four parts of this article, which are set up as individual papers, represent their class projects.

2. In-Class Exercises. I try to convince the students that good writing is important. This is done through exercises like the ones below. The exercises encourage the students to understand the contents of a text; identify its main ideas; analyze how the writing affects the reader and explain how this is achieved; and practice their own writing.

Exercise 1. Read the two summaries of the paper Rigid Body Dynamics with Friction and Impact by David Stewart [5]: The first one is the abstract of the paper [5, p. 3], and the second one an introduction to the paper by Nick Trefethen [6].

Does one summary do a better job of enticing you to read the paper, and why? If so, why does the other summary leave you indifferent?

Exercise 2. Test Your Writing IQ.

1. Question: What is the primary purpose of writing? (Answer in one word.) Answer: Communication.

2. Question: As a writer, to whom are you primarily obligated? (Answer in one or two words.)

Answer: Your readers.

3. Question: What does question 2 imply as far as the process of writing is concerned? (Answer in fewer than ten words.)

Answer: Always put yourself in the place of your readers.

Or: Write so that the readers can understand you.

Exercise 3. Questions to Students.

- Do you like writing? If not, why?

- Which types of writing do you enjoy the most, or the least?

- Do you see any advantage to writing (besides getting a degree or expanding your $C V)$ ?

- Have you found a strategy that makes writing easier for you? 
Exercise 4. Can you improve the two examples for opening paragraphs on page 88 in Nick Higham's book [1]? Think about removing possessive pronouns, shortening sentences, and delaying or avoiding the use of symbols.

Exercise 5. Read the mission statements on the web pages of SAMSI, the Statistical and Applied Mathematical Sciences Institute (http://www.samsi.info), and Sandia National Laboratories (http://www.sandia.gov/mission/index.html).

Write these mission statements in your own words (imagine that you are trying to explain them to a friend).

Exercise 6. Read section 2.5 about cancellation in Beresford Parlett's book The Symmetric Eigenvalue Problem [3]. Give a one-sentence explanation of catastrophic cancellation. Was this easy or difficult? Why?

After they have gone through these exercises, I hope that the students understand better why good writing is important and that they are motivated to put effort into their writing. Moreover, I hope that they have learned the need to identify their audience and to write with this audience in mind.

3. Student Presentations. The student presentations consist of rehearsals for research talks at conferences as well as for talks at the graduate student recruitment weekend. Each student gives at least one presentation, lasting between 10 and 30 minutes. All presentations use laptops.

Each presentation gets three runs. During the first run no questions or comments are allowed, so that the students can critique the talk as a whole. During the second run, the students critique each slide in detail. Then the presentation is revised, and a third run occurs during the next class meeting. We found that no more than three runs were needed.

The books by Higham [1, sections 10 and 11] and Krantz [2, section 4.4] provide plenty of good advice on how to prepare and give talks. The section Help with Presentations on the class web page, http://www.math.ncsu.edu/SIAM_Review_paper/ ma798I, contains additional tips. Below are examples of criteria we use to judge the different runs.

Criteria for evaluating the presentation as a whole after the first run include:

- Does the presentation carry a clear message?

- Is the exposition appropriate for the target audience?

- Does the presentation tell a story? Is the story exciting and coherent?

- Is the presentation well organized?

- Does the presentation start with an outline and finish with a summary?

- Does the presentation finish on time?

- Are the main ideas well explained by examples or pictures?

- Is the visual appearance of the presentation unhurried and calm? 
Criteria for evaluating individual slides during the second run include:

- Does the slide carry a clear message, preferably a single idea?

- Does the slide consist of at most 7-10 lines of text?

- Is the prose short and to the point?

- Are the mathematical expressions readable, intelligible, and as simple as possible?

- Is the notation mnemonic and intuitive?

- Is every mathematical expression accompanied by an interpretation (oral or visual)?

- Are all symbols defined?

- Are all subscripts and superscripts absolutely necessary?

- Do the coordinate axes in graphs have meaningful labels?

- Does the slide make effective use of colors (e.g., by attracting the eye to the important parts, or by connecting related entities or concepts with the same color)?

- Does the summary slide reiterate succinctly the main ideas of the talk?

- Is the transition from the current to the next slide smooth?

The third run is evaluated according to all criteria. When a student is giving a presentation, it is helpful to designate another student as note taker to record comments from the audience.

The students considered the presentations to be the most difficult part of the course, because they were being put on the spot and there was no room for error. In contrast, they found the writing easier because one can always make yet another revision.

4. Homework. The purpose of the assignments below is to introduce mathematics to nonmathematicians, to help the students understand and analyze exposition, and to prepare a CV. These assignments are interspersed with the work on the class project described in section 5 (hence the nonconsecutive numbering of homeworks below; the missing homeworks are listed in section 5). The due dates for the assignments vary from 2 to 7 days.

Homework 1A. Write a one-page introduction to the "business" of your field for outsiders who are unfamiliar with mathematics.

Put yourself in the shoes of a layperson: What do botanists or soil scientists or organic chemists do? Where do they work? What do they study? What kinds of questions do they ask? What kinds of methods do they use? Why is their research important? Who uses the results of this research? Who is affected by its results?

After the business descriptions have been handed in, I distribute them to everybody in class. 
Homework 2. For each business description from Homework 1A answer the following questions.

What are the good points? What needs improvement? Constructive suggestions are always appreciated! Describe your honest reaction to each submission: Did you find the description clear, lively, awkward, exciting, dry, pompous, confusing, stimulating, bewildering, entertaining, boring, irritating, vague, condescending, vapid, superficial, careless, clumsy, unrefined, muddled, suspenseful, precise, detached, incomprehensible, animated, inadequate, incoherent, impenetrable, inarticulate, ..., and why?

Homework 3. Read the paper On the Norm of Idempotent Operators in a Hilbert Space by Vladimir Racočević [4].

Determine the who, what, and why. (Who is my audience? What do I, the author, want to say? Why do I want to say it? See [2, sections 1.1 and 1.2].) Then write a single sentence that summarizes the contents of the paper. Make sure you understand the mathematical details.

We will discuss the following issues in class: Is the paper easy to read and understand? Why or why not? Was it easy to write the summary? Why or why not?

Homework 5. Revise your business description from Homework 1A, taking into account the responses from Homework 2, as well as my suggestions.

Homework 6. The paper "The Perfidious Polynomial" by Jim Wilkinson [7] won the Chauvenet prize of the MAA in 1987. The prize is awarded for an outstanding expository article; its recipients are listed in http://www.maa.org/Awards/ chauvent.html [sic].

1. For each section state the main idea conveyed in that section.

2. Write an abstract for the paper.

3. Express items (i), (ii), (iii) in section 4 of the paper in your own words.

4. Does the introduction prepare you for the remainder of the paper? Explain why or why not.

5. Describe how this paper differs from other papers you have read.

We will discuss the issues above in class.

Homework 13 asks the students to prepare a CV. In the class meeting preceding the assignment, I show the students samples of CVs, and we discuss the contents and layout of an effective (academic) CV.

Although the students considered the presentations to be the most difficult part of the course (due to their real-time nature and small margin for error), completing the writing assignments seemed to cause more frustration than preparing the presentations. One of the reasons is that suggestions for improving presentations are specific (e.g., put fewer lines on the slide, display important terms in red, make the symbols in a formula more mnemonic), hence easy to implement. In contrast, suggestions for improving writing are much less tangible (e.g., make it flow more smoothly, make it livelier), and it is not at all clear how to realize them. 
5. Class Project. This is the centerpiece of the course (represented by the four example papers). Individually, or as part of a group, each student writes a paper for a general mathematical audience.

The class project starts with the very first homework of the semester (Homework 1B below), so that the entire semester is available for revisions and the project has a good chance of being completed by the end of the semester.

Homework 1B. Submit a pre-proposal for your class project, and determine the main ingredients: authors (papers can be written individually or in groups), subject (preferably related to your research), and audience (SIAM Review, SIAM News, or American Mathematical Monthly).

The web page for Homework $1 \mathrm{~B}$ also provides links to the description of these journals and to instructions for authors. The students decided to write papers for SIAM Review about application problems that motivate their thesis research.

Work on the class project proceeds gradually, guided by a sequence of homework assignments. As a consequence, students are not overwhelmed by a huge writing assignment, looming large at the end of the semester, and they do not have much opportunity to procrastinate. Moreover, I am able to monitor their progress, keep the projects on track, and help those who are stuck.

The next assignment tries to preempt writer's block and encourage brain storming by first focusing on content rather than packaging.

Homework 4. Prepare a preliminary, informal pre-draft of your class project.

Here are some hints to get you going: Compile a table of contents. Sketch a rough outline of the story you want to tell in your paper. Write a couple of sentences for each section; jot down thoughts, key words, or issues you want to address. Draw a picture of your example. Collect papers you need to reference. For whom are you writing the paper-who is your audience? What are you planning to say? Why are you saying it? If you are having difficulty getting started, think about the following:

I am going to tell you about...

It's really important because...

Let me give you a concrete example...

Here is where it occurs specifically...

It's interesting because...

It has defeated scientists so far because of...

The mathematical difficulties are...

People have tried the following approaches...

But they failed because of...

Then someone came along and tried...

The submissions for Homework 4 were extremely ambitious, almost encyclopedic - quite appropriate for book proposals, actually. The drafts contained too much material and lacked focus. They were geared towards an audience of experts, rather than a general mathematics audience (apparently the journal descriptions and instructions for authors did not hit home the first time around).

The following assignment tries to remedy this situation by narrowing down the contents and focusing on the target audience. 
Homework 7. Write a first draft.

1. Describe a single application, experiment, or problem.

Describe the "physical" situation or setup. Include graphics, if possible. Tell the reader what $\mathrm{s} / \mathrm{he}$ is seeing in the picture.

Explain why this example is important, difficult, representative, or surprising.

2. Present the mathematics.

Distill it to one or two central ideas you would like readers to understand. Simply write up these one or two ideas. Explain notation, but use as little as possible. Provide plenty of intuition. Explain symbols and expressions in equations. Explain meanings of variables. Interpret derivatives, perhaps as rates of change or acceleration.

3. Find an inspiring title for your paper.

Two revisions (Homeworks 8 and 10) and one peer critique (Homework 9) follow. The papers resulting from the revision in Homework 10 are distributed to everybody in class.

Homework 11. Review each paper to determine whether it is ready for publication. If not, justify why and submit your reasons in writing.

Consider the following criteria:

- Is the paper consistent with the editorial objectives?

See the Editorial Policy for SIAM Review (http://www.siam.org/ journals/sirev/policy.php) and the Instructions for Authors (http://www. siam.org/journals/sirev/authors.php).

- Is the presentation clear and well organized?

- Is the notation well conceived and consistent?

- Are the references relevant and complete?

- Does the title accurately describe the paper?

Now comes the next-to-last revision and the writing of the abstract.

Homework 12.

- Revise your paper according to the suggestions from Homework 11.

- Write a brief abstract for your paper (that is, summarize its contents briefly without being vague).

- List key words and AMS subject classifications (http://www.ams. org $/ \mathrm{msc} /$ ).

The purpose of the last assignment below is to create a distance between the students and their work, so that afterwards they are again able to see the forest for the trees.

Homework 14. Do not look at your paper for two weeks.

Then read your paper, and only afterwards read my comments. Make the necessary revisions, so that your paper is ready for publication. 
6. Outcome. The four parts that follow represent the final versions of the class project. All papers were completed on time and submitted to SIAM on the last day of classes.

The last class meeting was conducted at Cup a Joe's, a local coffee house, where we evaluated the course. The students found the assignments and in-class presentations helpful. A unanimous complaint was that too much time was spent on mathematics (to understand the papers from Homework 3 and 6); and that this time could have been better spent on learning to write abstracts for talks, Ph.D. theses, or proposals to funding agencies.

There are many other assignments that could be included in a course like this. For instance:

- Abstracts and summaries for talks, papers, Ph.D. theses, or proposals to funding agencies.

- A review of a paper for MathSciNet (http://www.ams.org/mathscinet). The students have to extract the main ideas of a paper and describe them succinctly and clearly.

- A referee's report for a journal paper. The students learn about criteria for evaluating papers, as well as the format and style of referees' reports.

- A book review (or a comparison) of the textbooks. By the end of the semester the students are thoroughly familiar with the texts by Higham and Krantz (because all homework includes specific reading assignments), putting them in a position to write an informed review (section 4.2 in Krantz's book contains advice for book reviews).

- More detailed feedback for presentations. When a student gives a presentation, the other students take notes about the presentation, polish these notes at home, and then give them to the presenter. The notes tell the presenter which messages from the talk came across and which ones did not.

\title{
Part A. The Evolution of an Optimization Test Problem: From Motivation to Implementation. By Daniel E. Finkel and Jill P. Reese.
}

\begin{abstract}
We introduce a water-supply problem considered by the optimization and hydrology communities for benchmarking purposes. The objective is to drill five wells so that the cost of pumping water out of the ground is minimized. Using the implicit filtering optimization algorithm to locate the wells, we save approximately $\$ 2,500$ over the cost of a given initial well configuration.
\end{abstract}

Key words. optimization, implicit filtering, well field design, groundwater flow

AMS subject classifications. $93 \mathrm{C} 20,78 \mathrm{M} 50$

A.I. Introduction. Hydrologists and environmental engineers are often faced with the daunting task of choosing the appropriate optimization strategy for complex problems. In 2002, with this thought in mind, a series of groundwater test problems was proposed in $[14,15]$. This suite of test cases, known collectively as the community problems, is a set of prepackaged codes, including a preset initial condition, used to compare the performance of optimization algorithms. This collaborative research between engineers and optimization experts has led to an increased understanding of the advantages and disadvantages of current optimization algorithms, and helped to further optimization as a field. 
In this paper, we focus on one of the community problems and its evolution from fundamental equations to a well-defined optimization problem. We describe some of the modeling issues that arose during formulation and present the results obtained by the implicit filtering optimization algorithm.

A.2. Optimization. We focus on a particular community problem to illustrate some of the concepts and difficulties underlying the test cases. The objective is to find the optimal locations at which to drill five wells in an underground water-bearing region (an aquifer). Since the cost to install five wells is fixed at approximately $\$ 100,000$, the goal is to minimize the cost to operate the wells. Some regions underground contain more water than others, and a well located in a drier part of an aquifer has to work harder than a well located in a region of the aquifer where more water is available. Since the cost of pumping a well depends on its location within the aquifer, we want to find the $\vec{x}=(x, y, z)$ locations to drill the wells within the domain (aquifer) in order to minimize the cost of operation. Section A.3 explains this idea in more detail.

A.2.I. Test Problem Specifications. We consider an aquifer 1000 meters long on each side, 30 meters deep, and of homogeneous composition. Specifying homogeneous composition implies that all aquifer material properties are constant in both space and time. We assume that all five wells remove water at the same fixed pumping rate, $\mathcal{P}=-0.0064$ meters per second (we follow convention and indicate extraction by $\mathcal{P}<0$ ), and extract water at the same depth, $z_{w}$. The decision variables are thus reduced to $(x, y)$ locations within the domain.

A.2.2. Objective Function. The objective function of the minimization problem quantifies the intuitive relationship between the amount of water in the domain at a given location and the work required to pump water from that location. We think of $h_{i}$ as the amount of water available at well $i$, although more specifically it is the hydraulic head level at well $i$. The physical meaning of the hydraulic head level is discussed in section A.3. Given a pumping rate $\mathcal{P}^{y}$ in meters per year, ${ }^{1}$ and the 30-meter-deep aquifer, the operating cost per year for well $i$ is given by

$$
f_{i}=\left(2.9 \cdot 10^{-4}\right)\left(h_{i}-30\right) \mathcal{P}^{y} .
$$

In (A.1), the constant $\left(2.9 \cdot 10^{-4}\right)$ is a cost coefficient provided in [14]. Since the pumping rate, $\mathcal{P}^{y}$, is negative the minimization problem is attracted to regions where the hydraulic head level, $h_{i}$, is large. However, Figure A.1 illustrates that if head levels exceed 30 meters, then water would flow over the ground surface and result in flooded land. Thus, (A.1) is formulated in conjunction with a constraint on the hydraulic head levels over the entire domain (including at the wells) such that

$$
10 \leq h \leq 30 .
$$

Just as keeping hydraulic head levels below 30 meters prevents flooding of the land, constraining the head levels to be greater than 10 meters avoids the possibility of pumping the land dry.

A.3. Groundwater Flow. From (A.1) we see that given the fixed pumping rate, $\mathcal{P}^{y}$, the only unknown in the objective function is the hydraulic head level at each

\footnotetext{
${ }^{1} \mathcal{P}^{y}$ is equivalent to the previously defined $\mathcal{P}$, although the units have changed. The units of $\mathcal{P}^{y}$ are meters per year, while $\mathcal{P}$ has units of meters per second.
} 


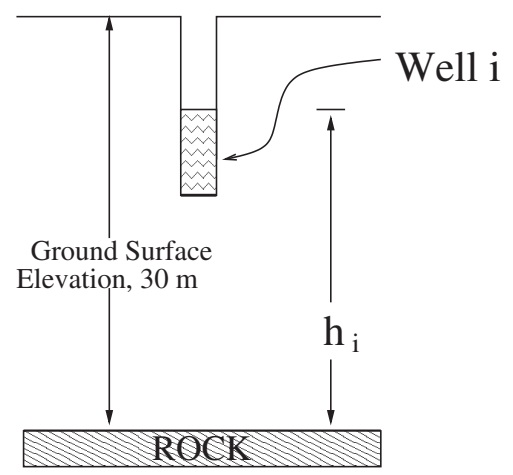

Fig. A.I Schematic of the cross section of a well in an aquifer.

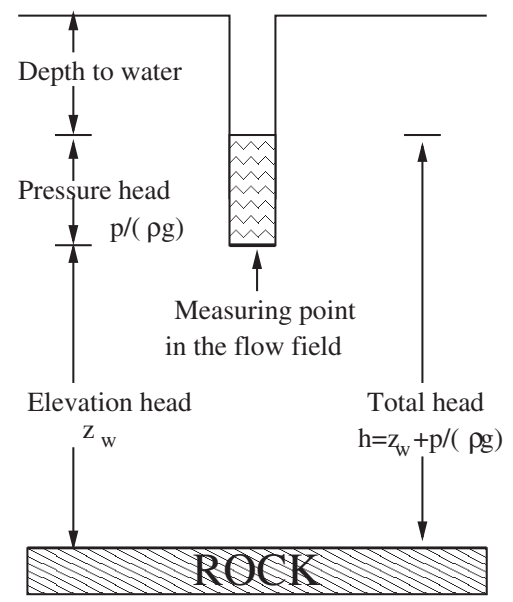

Fig. A.2 Diagram showing the components of hydraulic head (adapted from [9, p. 35]).

well. Computing the hydraulic head level requires a simulation involving the solution of a partial differential equation describing transient, three-dimensional flow through an aquifer that is entirely saturated with water. The simulation models how the installation and operation of wells in the aquifer alters the natural hydraulic head levels and flow pattern.

A pumping well causes a drop in pressure, $p$, within the aquifer close to the well. A pressure difference is the primary driving force for groundwater flow, so this pressure drop causes water to flow toward the well from areas of higher pressure. Other contributions to groundwater flow come from gravity, $g$; water density, $\rho$; and the well depth, $z_{w}$. Hydrologists combine the contributions of $p, g, \rho$, and $z_{w}$ into a single quantity known as hydraulic head. The hydraulic head level, $h$, can be thought of as the height to which the surrounding aquifer pressure forces water to rise at a given point in the domain, as shown in Figure A.2.

How the hydraulic head level, $h$, changes in time primarily depends on (i) how it changes in space, (ii) the rate at which the wells are pumping, and (iii) how easily 
the water can move through the soil. The ease with which water can move through soil is expressed in a soil property called hydraulic conductivity, denoted here by the constant $H_{c}$. Another factor affecting the hydraulic head level is $C$, which accounts for the compressibility of both the water and the porous medium. Compressibility describes how much the volume of water changes in response to a change in pressure, and for these computations we assume slightly compressible flow with $C=10^{-6}$. The diffusion equation [11, p. 65]

$$
C \frac{\partial h}{\partial t}=H_{c} \nabla^{2} h+\mathcal{S}
$$

describes the three-dimensional flow of water through a homogeneous, isotropic domain, where the left side represents the rate of change in head level at a point in the domain. This rate of change is balanced by spatial changes in head levels due to diffusion and any external sources of hydraulic head, denoted by $\mathcal{S}$. For our test problem the only contribution to $\mathcal{S}$ comes from the five pumping wells, and the mathematical representation of $\mathcal{S}$ depends on how one chooses to model the wells.

A.4. Simulation. The hydraulic head levels are calculated by a computer simulation of the aquifer using MODFLOW software from the U.S. Geological Survey [12]. MODFLOW inputs include the well locations and pumping rates, initial hydraulic head levels throughout the domain, boundary conditions on hydraulic head, and properties of the domain. The software uses a point-source well model; thus, the MODFLOW-specific mathematical representation for $\mathcal{S}$ is

$$
\mathcal{S}=\mathcal{P} \sum_{i=1}^{5} \delta\left(\vec{x}-\vec{x}_{i}\right),
$$

assuming that well $i$ is located at the point $\vec{x}_{i}, \vec{x}$ is a generic point within the aquifer, and $\delta$ represents the Dirac delta function. The software outputs the hydraulic head level at each point in the domain. The simulation accounts for groundwater flow in all three spatial dimensions; however, the plots in this paper show only two-dimensional slices of the domain.

The initial hydraulic head levels, $h(x, y, z, t)=h\left(x, y, z_{w}, 0\right)$ (given in meters), that were input to MODFLOW are shown by the contour lines in Figure A.3(a). This initial condition is included in the community problems package. Figure A.3(a) illustrates that the highest hydraulic head levels occur at the lower left corner of our domain, while the lowest head levels are in the upper right corner. Like pressure, hydraulic head provides a potential which induces flow, so initially the groundwater is flowing from lower left to upper right. This is illustrated by the velocity field which overlays the contour plot.

The boundary conditions and aquifer properties required for this simulation are provided in $[14,15]$.

A.5. Constraints. To pose the optimization problem in a physically reasonable fashion, constraints are introduced in addition to those on hydraulic head level (see $($ A.2)). The $(x, y)$ locations of the five wells are constrained so that (i) the wells are not too close to the boundary of the domain, and (ii) the wells are not too close to each other. These conditions prevent us from pumping water from our neighbor's property and from installing two wells in the same location; moreover, they add difficulty to the optimization problem. 


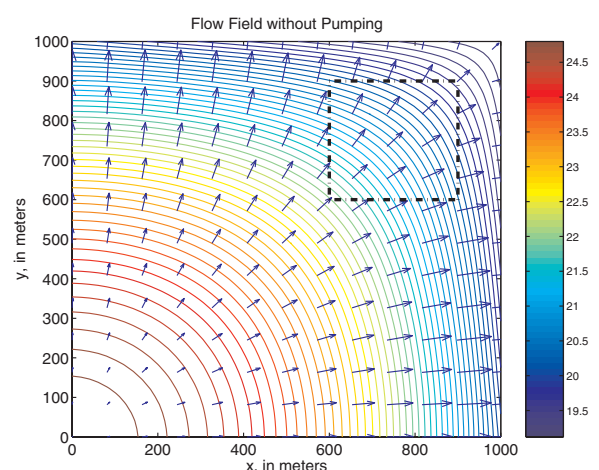

(a)

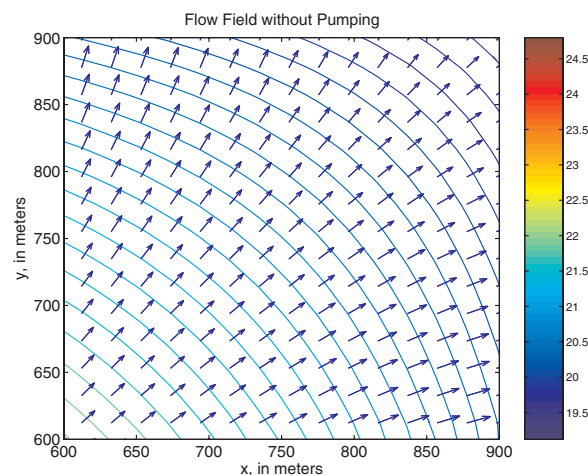

(b)

Fig. A.3 Hydraulic head contours (in meters) when the wells are installed but not pumping. (a) Entire domain. (b) Enlarged view of the region within the black dotted square.

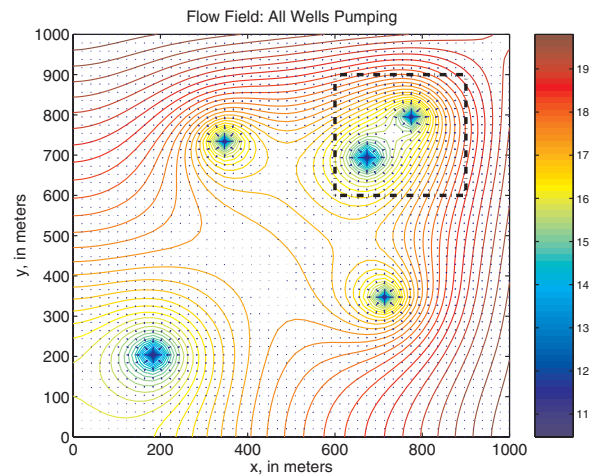

(a)

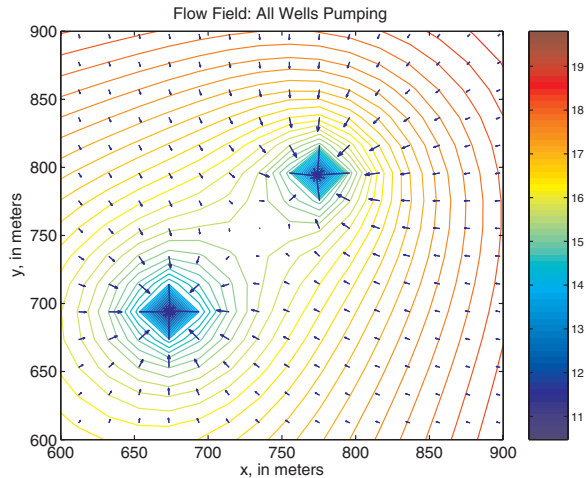

(b)

Fig. A.4 Change in head levels when the wells pump in the initial configuration included in the prepackaged code. The contours form blue diamonds near the wells due to the pressure drop at an extraction well. (a) Entire domain. (b) Enlarged view of the region within the black dotted square.

Figure A.4(a) shows an initial iterate (well configuration) for the optimization problem that satisfies all the constraints. The contour plot shows how this well configuration affects the flow field once the wells begin pumping. Figure A.4(b) is an enlarged view of a portion of the domain and shows that the water is flowing toward each well (because of a drop in pressure). As a comparison, Figure A.3(b) shows the same part of the domain when no wells are pumping. Note that the head levels over the whole domain are lower once the wells begin pumping water out of the domain.

A.6. Optimization Problem. Again, the objective is to minimize the cost of supplying water for five years by optimally placing five wells in a given region. We can find the total cost for operating all the wells by adding the individual costs to operate each well, and then multiplying by how long they are in operation (five years). The total cost is

$$
f=5 \sum_{i=1}^{5} f_{i},
$$




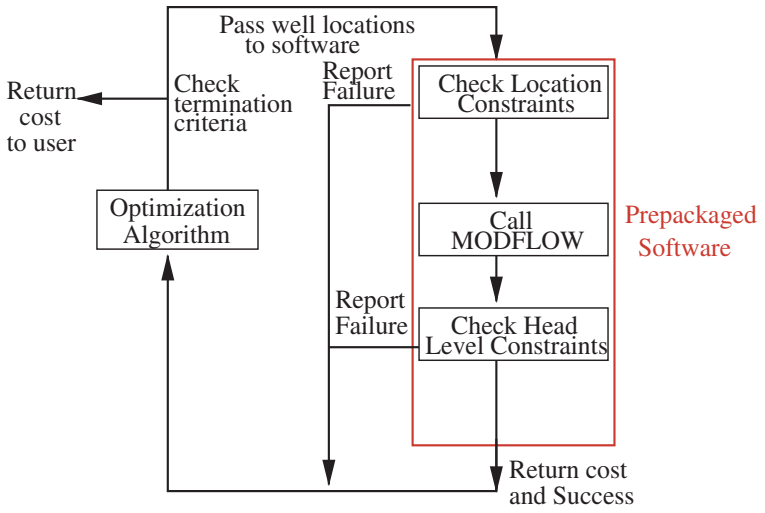

Fig. A.5 The relationship between the optimizer, the prepackaged code, and the MODFLOW simulator.

Table A.I Cost comparison between initial condition and optimal solution obtained using IFFCO.

\begin{tabular}{|c|c|}
\hline Initial cost & $\$ 26,959$ \\
\hline Optimal cost & $\$ 24,314$ \\
\hline Percent decrease & $10.9 \%$ \\
\hline Simulator calls & 273 \\
\hline
\end{tabular}

where $f_{i}$ is defined in (A.1). The constraints described in the previous section are quantified and described in [10]. The five well locations are passed as input to the objective function. Before running the MODFLOW simulation, the well locations are checked within the objective function to verify that the wells are not too close to each other. If this constraint is satisfied, MODFLOW is called with the given well locations as input. The simulator calculates the hydraulic head level at each point in the domain. These head values are then checked within the objective function to ensure that they satisfy (A.2). Figure A.5 describes the optimization procedure.

MODFLOW is computationally expensive; thus, it accounts for much of the time required to compute the objective function. In [10], several optimization algorithms are tested on this problem and their results compared by determining how many calls to the simulator were necessary to obtain an optimal solution.

A.7. Results. The results presented in this section are computed with the implicit filtering algorithm. Implicit filtering is a finite-difference quasi-Newton method for solving noisy optimization problems which requires a feasible initial iterate $[8,13]$. Implicit filtering was chosen as a representative case for the results collected in [10], as it was the first algorithm applied to the community problems. Furthermore, example results from a particular implementation of the implicit filtering algorithm, IFFCO [8], are included in the community problems distribution.

The initial well locations are shown in Figure A.3(b), corresponding to an operational cost of $\$ 26,959$. IFFCO reduces the operational cost to $\$ 24,314$, a savings of $10.9 \%$. The optimization process involves 273 evaluations of the objective function. See Table A.1. The final well locations are shown in Figure A.6, while Figure A.7 presents the optimization history for this problem. This history shows the decrease in cost at each iteration. 


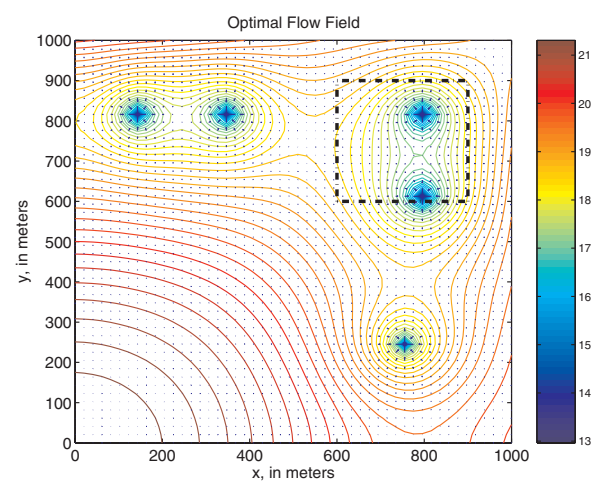

(a)

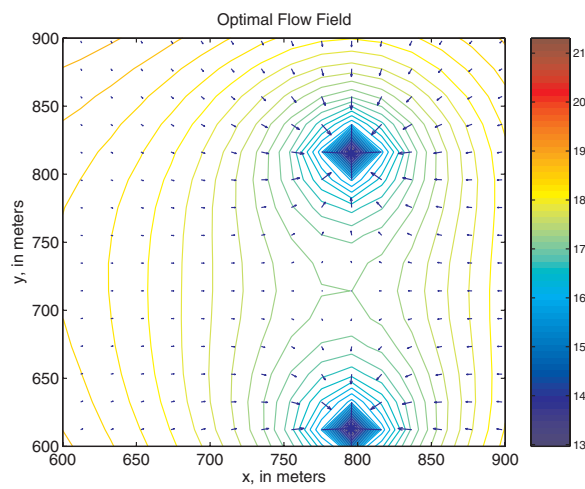

(b)

Fig. A.6 Optimal well configuration. (a) Entire domain. (b) Enlarged view of the region within the black dotted square.

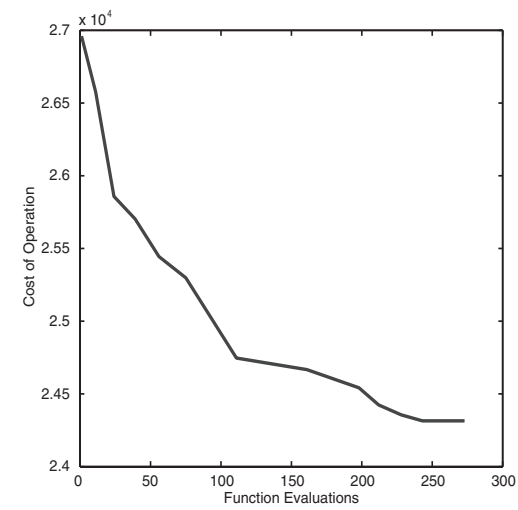

Fig. A.7 Reduction in operational cost as a function of number of calls to the objective function.

A.8. Summary. Collaboration between mathematicians and environmental engineers has led to the creation of a suite of community groundwater test problems. These test problems afford environmental engineers the ability to compare groundwater flow simulators, and mathematicians a means to evaluate optimization algorithms.

Determining the cost of extracting water from the ground via a well requires a fundamental understanding of the flow of water through an aquifer. Equation (A.3) is the basis of the optimization problem; its unknown, the hydraulic head level, is used to calculate the cost of operating an extraction well.

Although we report the results of only one optimization package here, seven other software packages were applied to this problem and the results are compiled in [10]. One of the goals of this project is to better understand which optimization algorithms should be used to solve groundwater flow problems, and how to pose these problems to efficiently find optimal solutions. Further information regarding the community problems can be found on the website http://www4.ncsu.edu/eos/users/c/ctkelley/www/ community.html. 
Part B. Finding the Volume of a Powder from a Single Surface Height Measurement. By Christopher Kuster.

\begin{abstract}
The volume of powder poured into a bin with obstructions is found by calculating the height of the surface at every point. This is done using the fast marching algorithm. We look at two different bin geometries and determine the volumes as a function of the powder height under the spout. The surface of the powder satisfies a two-dimensional eikonal equation. This equation is solved using the fast marching method.
\end{abstract}

Key words. granular materials, sand piles, eikonal equation, fast marching

AMS subject classifications. $70 \mathrm{H} 20,00 \mathrm{~A} 69,35-01$

B.I. Introduction. In the pharmaceutical industry, many ingredients are in powder form. These powders are stored in bins before they are combined to form pills. To guarantee the same amount of each ingredient per pill, the bins contain solid inserts that keep the flow of powder constant (see Figure B.1). It is important to keep track of how much powdered ingredient there is in each bin. Knowing the amount of powder in a bin allows for a quick check on the flow rate and helps ensure that the drugs are not being skimmed off for illegal sale. Our goal is to accurately measure the amount of powder in a bin using only observations of the surface. To this end, we create a mathematical model of the powder and then employ existing numerical methods to accurately solve for the height of the surface at every point in the bin. Once the height of the surface is known, the volume of powder in the bin is just the volume under the surface.

If the powder is put into the bin slowly from a single source (say, a spout somewhere above the bin), then the only physical parameter necessary to model the surface is the angle of repose (see Figure B.2). This is the maximum slope the surface of the powder can have without causing an avalanche. Every granular material has its own angle of repose that depends on the average shape and roughness of the individual grains. For the purposes of our model, we assume that at each point, the largest angle that the surface makes to the ground is equal to the angle of repose. This is a simplified version of the model used by Aronsson [17].

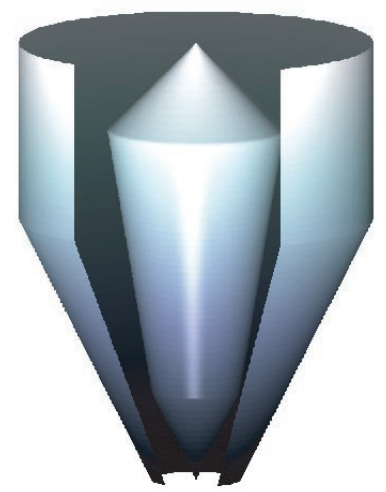

Fig. B.I Cutaway view of a bin with a flow correction insert. 

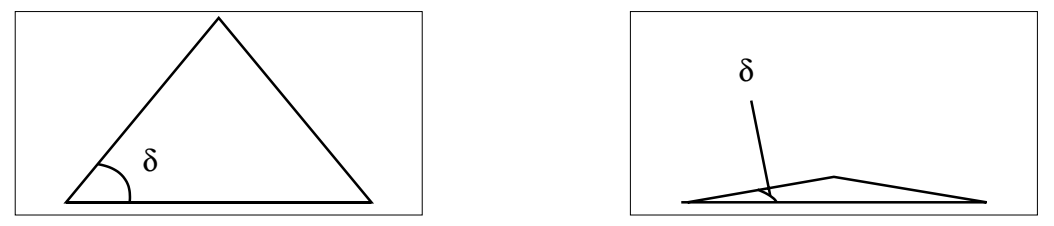

Fig. B.2 Left: Large angle of repose. Right: Small angle of repose.
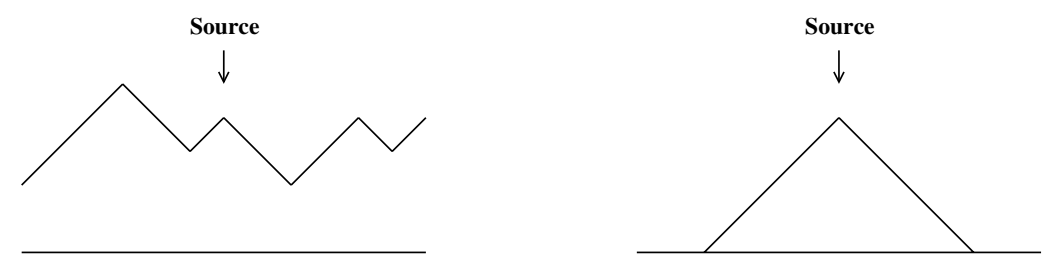

Fig. B.3 Possible solutions to (B.6).

If we define $f(x)$ to be the height of the powder at a point $x$, and $\delta$ to be the angle of repose, then in one dimension our assumption leads to the equation

$$
\left|f^{\prime}(x)\right|=\tan \delta .
$$

We have used the fact that, by definition, the derivative is the same as the slope, which is in turn equal to the tangent of the angle of repose. Requiring (B.6) to be satisfied nearly everywhere leads to an infinite number of solutions for the surface of the powder, $f(x)$ (see Figure B.3 (left)). Physically, the height of the surface decreases farther from the source, so we add that condition to the model. This leads to a single solution [19] (see Figure B.3 (right)).

In contrast to the one-dimensional case, in higher dimensions we can model obstacles that cannot be penetrated by the powder. Inside obstacles, we define the angle of repose to be $90^{\circ}$. This means that the slope is infinite and the height of the powder is zero inside the obstacle [16]. This leads to a discontinuous function $S(x, y, z)$, defined by

$$
S(x, y, z)= \begin{cases}\tan \delta & \text { if }(x, y, z) \notin \text { Obstacle } \\ \infty & \text { if }(x, y, z) \in \text { Obstacle }\end{cases}
$$

The function $S$ is defined over all space, but since we only are looking at the surface, (B.6) can be generalized to the two-dimensional eikonal equation

$$
|\nabla f(x, y)|=S(x, y, f(x, y)) .
$$

Notice that the value of $S$ on the right-hand side of the equation depends on the solution, $f$. The word eikonal comes from the Greek word for image, and (B.7) has long been studied in the context of optics [18].

B.2. Numerical Methods. A standard method for solving the eikonal equation is the fast marching method first proposed by Tsitsiklis in 1994 [21]. The basic idea of the fast marching method is that it calculates the solution along contour lines moving outward from the source. Given a single measurement of the height at the source, this method automatically returns a solution that satisfies the condition of height decreasing with distance from the source (avoiding obstacles). A detailed description of the fast marching method is given by Sethian [20]. 

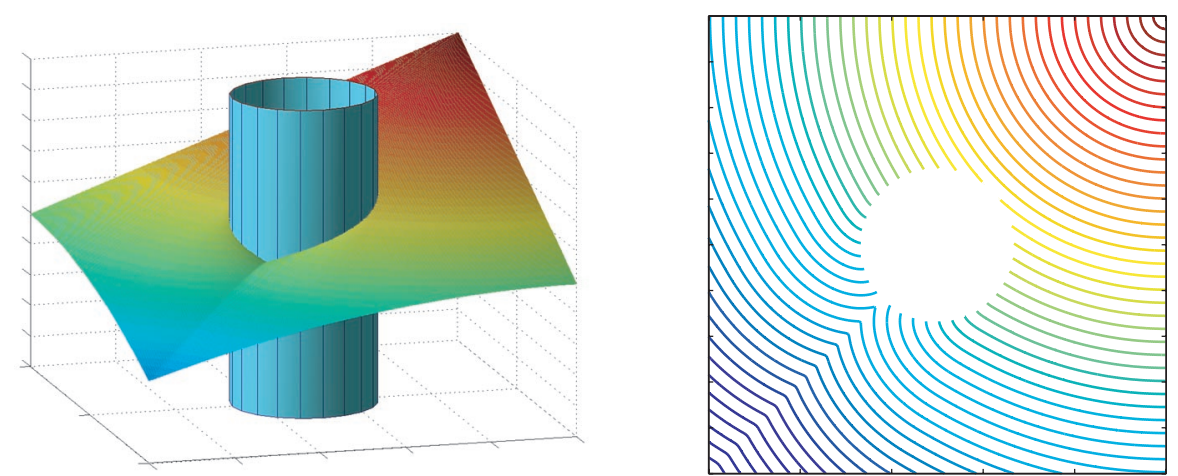

Fig. B.4 Surface profile around a circular prism (red is high, blue is low). Left: surface. Right: contours.

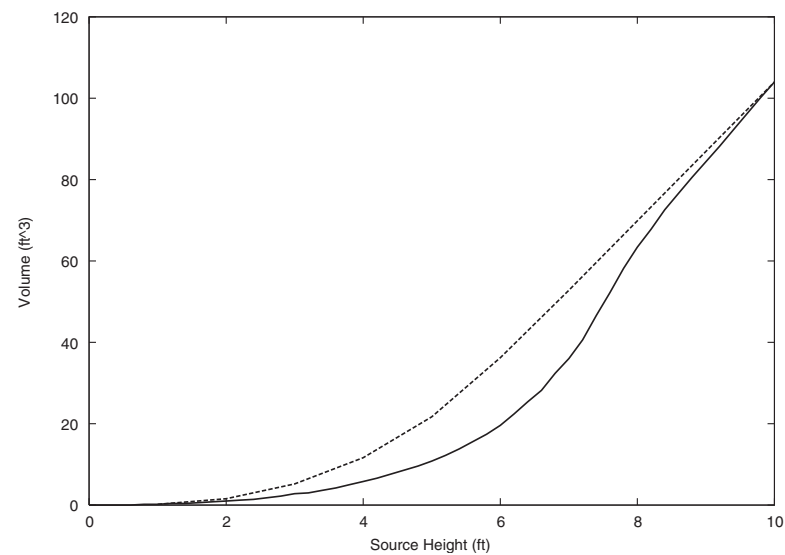

Fig. B.5 Volume as a function of height. Solid: Results from Figure B.1. Dashed: Results from Figure B.6.

B.3. Results. First, we tested the algorithm on a square domain containing a cylindrical obstacle. The results are shown in Figure B.4. The left image shows the surface of the powder and the cylindrical obstacle. The right image is a plot of the height contours as seen from directly above the obstacle. The source is directly above the highest point. Near the source, the surface contours are concentric (quarter) circles indicating that the solution there is a cone. Opposite the source, however, the obstacle causes a valley. This is also observed in physical experiments.

Finally, we apply the fast marching method to the case of the realistic bin shown in Figure B.1. We estimate the volume of powder in the bin by integrating over the surface. In Figure B.5, the computed volume is shown as a function of the height at the source. The results for a square bin with a cylindrical obstacle (Figure B.6) are also shown for comparison. The cylinder was chosen so that the volumes are equal when the height at the source is 10 (at the right side of Figure B.5). The volume of powder in the case with the cylinder (dashed line) increases smoothly with the height at the source. In fact, once the entire floor of the square bin is covered, the volume increases linearly with changes in height. In the case of the more realistic bin (solid 


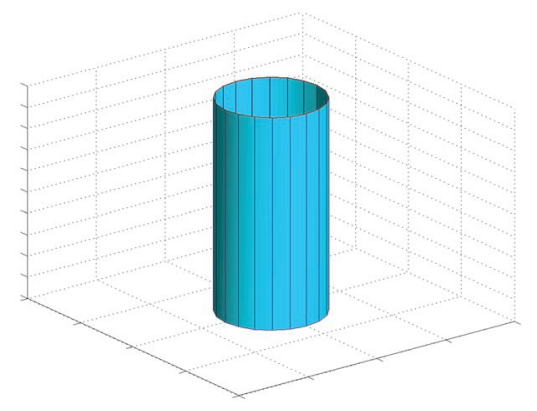

Fig. B.6 Cutaway of square bin with cylindrical obstacle.

line), changes in the shape of the walls and the obstacle lead to the volume being a more complicated function of the height at the source.

B.4. Summary. We start with a single measurement of the height of the powder surface under the spout. Then, using a single physical parameter (the angle of repose) to model the surface of a powder, we calculate the height of the surface at every point. With this information, we are able to estimate the volume of powder in the bin. The results we obtain correspond to those expected. More details about this method are available in [16].

\section{Part C. Finding Oscillations in Resonant Tunneling Diodes. By Matthew Lasater.}

Abstract. Resonant tunneling diodes (RTDs) are ultrasmall semiconductor devices that have potential as very high-frequency oscillators. To describe the electron transport within these devices, physicists use the Wigner-Poisson equations which incorporate quantum mechanics to describe the distribution of electrons within the RTD. Continuation methods are employed to determine the steady-state electron distributions as a function of the voltage difference across the device. These simulations predict the operating state of the RTD under different applied voltages and will be a tool to help physicists understand how changing the voltage applied to the device leads to the development of current oscillations.

Key words. Wigner-Poisson equations, continuation methods, Hopf bifurcation

AMS subject classifications. $81 \mathrm{~S} 30,35 \mathrm{~B} 32$

C.I. Introduction. When consumers choose new electronic devices, one important factor is the size of the device. Whether getting a new cell phone or buying a laptop, most people want a smaller, more compact device. Through technological advances electronic devices are becoming ever smaller. Current research on electronic devices involves analyzing their operation at the nanoscale level $\left(10^{-9}\right.$ meters), which is the typical length scale of molecules. At this tiny length scale the device physics are determined by quantum mechanics instead of classical electromagnetism. One such nanoscale device being investigated is the resonant tunneling diode (RTD).

In the 1980s, scientists and engineers began using RTDs in circuits in an attempt to produce a high-frequency power source $[29,28]$. High frequency in this case means terahertz $(\mathrm{THz})$, which is $10^{12}$ oscillations per second and 1000 times faster than current computer processors. Small devices that can produce and sustain $\mathrm{THz}$ 


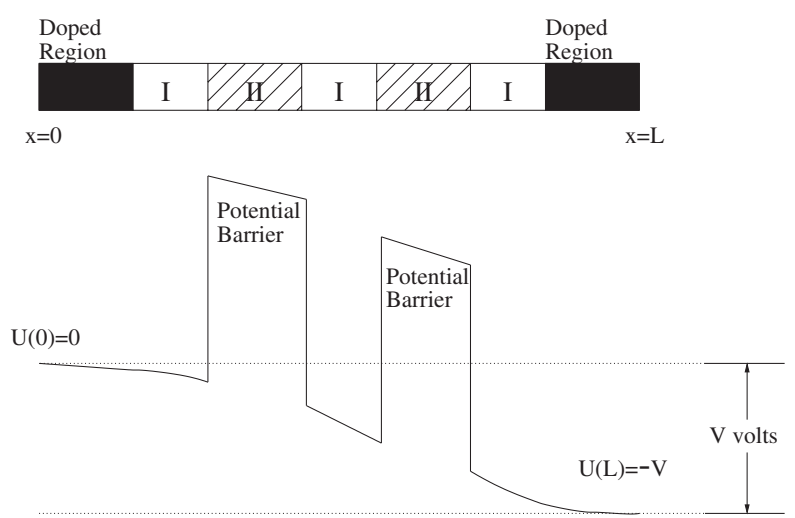

Fig. C.I Diagram of an RTD and its electric potential $U$.

oscillations are of considerable interest to the U.S. government for their potential military applications. One such application is the remote detection of a biological weapon in a battlefield environment. Research on identifying biological agents with $\mathrm{THz}$ radiation is being conducted [25]. The energy in the $\mathrm{THz}$ radiation excites the molecules in biological agents, making it possible to use $\mathrm{THz}$ radiation to determine the molecular structure of biological agents. Small devices that can act as $\mathrm{THz}$ radiation source/receivers would be required if this research is successful since the mobile environment of warfare demands compact devices.

While $\mathrm{THz}$ oscillations exist in the above-mentioned circuits, unwanted lower frequency oscillations also develop. These lower frequency modes result in a power loss which makes these circuits impractical. Ongoing RTD research involves theoretically analyzing the RTD outside of a circuit to see if high-frequency oscillations can be produced and sustained in the RTD itself [31]. The research is theoretical since no physical experiments are performed, only computer simulations. In the simulation the RTD is isolated from the circuit so that external effects are excluded. A voltage difference is applied across the RTD which creates a current. Engineers and scientists want to know which voltages give rise to current oscillations in the RTD.

C.2. RTDs. The top of Figure C.1 shows a diagram of an RTD. An RTD is created by joining together two different semiconductors, type I and type II. Two regions of type II semiconductor are placed between three areas of type I. Far to the left and right of the type II regions, the semiconductor is doped (represented by the dark area). In the doped regions atoms containing more (or fewer) electrons than the semiconductor itself are inserted to add (or remove) extra electrons.

The bottom of Figure C.1 shows the electric potential in an RTD. The electric potential is made up of two parts. One part is the electrostatic potential created by the electrons, the smooth portion of the electric potential. The other part represents the two potential barriers that arise since the device is a composite of two different semiconductor materials.

In Figure C.1 a voltage drop of $V$ volts attracts electrons to the right side. The far left of the RTD, from where the electrons are moving, is called the emitter, and the far right of the RTD, to where the electrons are moving, is called the collector. The part of the RTD that is between the two potential barriers is called the main quantum well. Classically, an electron is treated as a particle. If a particle runs into a potential 


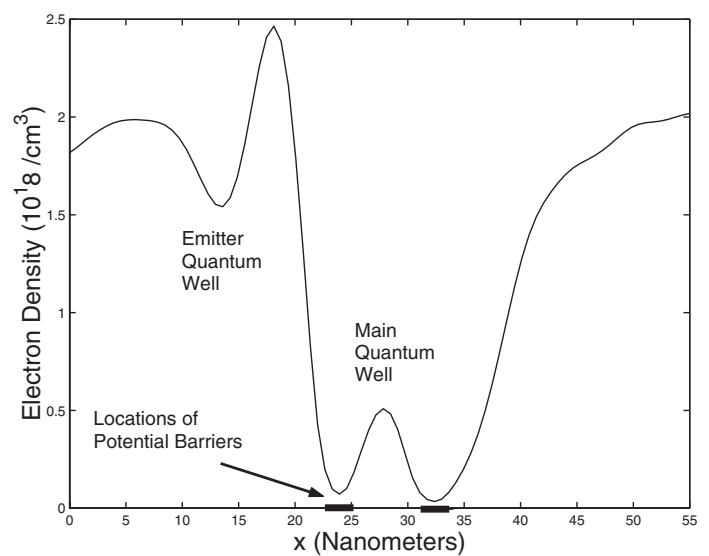

Fig. C.2 Electron density in RTD.

barrier, it is reflected back if it does not have enough speed to overcome the barrier. In quantum mechanics, though, electrons are treated as waves, and no matter how slowly the electrons are traveling, they still have some probability of passing through the barriers. This effect is known as quantum tunneling, and it is the basic idea behind the device.

The electron waves that move toward the left barrier from $x=0$ (called the incidental waves) either (1) transmit through the barrier or (2) reflect back to $x=0$. How many waves transmit through the barrier depends on the voltage difference $V$. If many electron waves are reflected back from the barrier, a cancellation between the incidental waves and the reflected waves occurs. This effect is known as quantum interference. Quantum interference creates an area to the left of the barriers with a depletion of electrons. This area is referred to as the emitter quantum well.

Figure C.2 shows the electron density (number of electrons per volume) across the RTD for a voltage difference that creates an emitter quantum well. In Figure C.2, the dark lines on the $x$-axis show the locations of the potential barriers. The electron density is the lowest at the potential barriers since the electric potential is so large in these regions. There is a local maximum in the electron distribution between the two potential barriers. This is the main quantum well and is where some electrons get "trapped" between the barriers. The recent theory for explaining the development of current oscillations postulates that the interactions between the emitter quantum well and the main quantum well are responsible for the oscillations [32].

Computer simulations are used to validate this theory and to gain a better understanding of the coupling between the quantum wells. Once this mechanism is understood, physical parameters in the simulations (heights of barriers, widths of barriers, widths of doped regions, etc.) will be varied to find the values that induce and sustain the current oscillations.

C.3. Mathematical Model. We introduce some mathematical techniques that give a better understanding of how an RTD operates. The first part presents the mathematical model for the time-evolution of the electrons in the RTD. The second part introduces continuation methods and their role in this model. The final part of this section explains the stability of a steady-state solution and how this helps to predict oscillations. 
C.3.I. Wigner-Poisson Equations. We model the electron transport in the RTD with the Wigner-Poisson equations [30]. They describe the distribution of the electrons in the device, which we denote by $f$. The distribution $f$ depends on the location of the electrons, the velocity of the electrons, and time. The Wigner-Poisson equations describe the time evolution, $\frac{\partial f}{\partial t}$, as a nonlinear function of $f$ and the voltage difference $V$ across the device. The Wigner-Poisson equations can be represented as $\frac{\partial f}{\partial t}=W(f, V)$.

For a given voltage difference $V$, we want to determine the current produced by the RTD. To this end we want to find the steady-state distribution of the electrons and use it to calculate the current output. The steady-state distribution describes how the electrons adjust themselves to the applied voltage $V$. For a given $V$, we want to find a distribution $f$ such that $\frac{\partial f}{\partial t}=W(f, V)=0$. Solving $W(f, V)=0$ for $f$ amounts to solving a nonlinear equation that depends on a parameter $V$. Continuation methods were devised to handle such a task.

C.3.2. Continuation Methods. Continuation methods solve nonlinear equations that depend on a parameter. In our case, the nonlinear equation is the time derivative of $f, W(f, V)$, and the parameter is the voltage difference $V$. Continuation methods find solutions, $f$, as a function of the parameter, $V$, represented by $f(V)$, such that $W(f(V), V)=0$. They trace the steady-state solution $f(V)$ as $V$ is varied. Once we have these steady-state solutions, we determine how the stability of these solutions changes as $V$ varies.

C.3.3. Stability of Steady-State Solutions. For our application, we are searching for voltage differences that render the steady-state distribution $f$ unstable. We want a specific kind of instability that creates oscillations about the steady-state distribution. In turn we have oscillations in the current of the RTD.

The stability of the steady-state solutions can be determined by computing $\frac{\partial W}{\partial f}$, the Jacobian of $W$. The Jacobian of $W$ measures how sensitive the time-derivative of $f, W(f, V)$, is to changes in $f$. It can be shown that if all the eigenvalues of $\frac{\partial W}{\partial f}$ at a steady state have negative real part, then the steady state is stable; however, if any eigenvalue has positive real part, then the steady state is unstable [22]. A point in the parameter space where the stability of the steady-state solutions changes is known as a bifurcation.

C.3.4. Hopf Bifurcation. The change in stability we are looking for is a (supercritical) Hopf bifurcation in which a steady state loses stability to a stable oscillatory solution. Instead of rigorously explaining how Hopf bifurcations arise, we present an ODE example to illustrate one.

Example I: Hopf Bifurcation. This example comes from [26]. We describe a two-dimensional state, $z=(x, y)$, by an ODE which depends on the real parameter $p$,

$$
\frac{d z}{d t}=g(z, p)=\left(\begin{array}{c}
p x-y-x\left(x^{2}+y^{2}\right) \\
x+p y-y\left(x^{2}+y^{2}\right)
\end{array}\right)
$$

The origin $(x, y)=(0,0)$ is a steady-state solution for any value of $p$ because $\frac{d z}{d t}=0$. The Jacobian of $g$ is

$$
\frac{\partial g}{\partial z}=\left(\begin{array}{cc}
p-3 x^{2}-y^{2} & -1-2 x y \\
1-2 x y & p-3 y^{2}-x^{2}
\end{array}\right)
$$




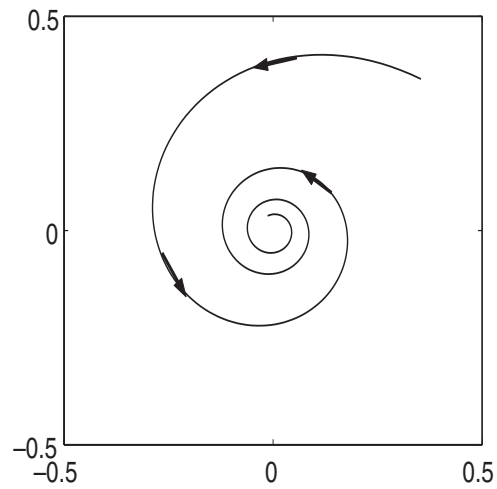

(a)

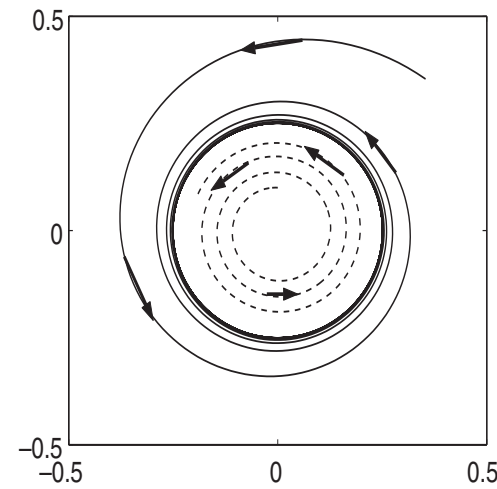

(b)

Fig. C.3 (a) Solution to ODE when $p<0$. (b) Solutions to ODE when $p>0$.

At the steady state $(x, y)=(0,0)$, the Jacobian of $g$ is

$$
\frac{\partial g}{\partial z}=\left(\begin{array}{cc}
p & -1 \\
1 & p
\end{array}\right)
$$

The eigenvalues of this matrix are $p \pm i$ where $i=\sqrt{-1}$; their real parts are $p$. For $p<0$ the origin is a stable equilibrium, for $p>0$ the origin is an unstable equilibrium, and $p=0$ is a bifurcation point in the parameter space. Figure C.3 shows typical solutions in $(x, y)$ coordinates for $p<0$ and for $p>0$.

If we switch from Cartesian coordinates $(x, y)$ to polar coordinates $(r, \theta)$, then we can examine the bifurcation point more carefully. Using the transformation $r=\sqrt{x^{2}+y^{2}}$ and $\tan (\theta)=\frac{x}{y}$, the ODE becomes

$$
\begin{aligned}
& \frac{d r}{d t}=r\left(p-r^{2}\right), \\
& \frac{d \theta}{d t}=1 .
\end{aligned}
$$

Case 1: $p<0$. If $p<0$, then $\frac{d r}{d t}<0$ for all $r>0$. Since $r$ is decreasing, all solutions approach $r=0$ (the origin). A typical solution is shown in Figure C.3(a).

Case 2: $p>0$. If $p>0$, in addition to our equilibrium at $r=0$, we find another nonnegative value of $r$ at which $\frac{d r}{d t}=0$, namely, $r=\sqrt{p}$. We look at three different regions of $r$-space to see what happens.

Region 1: $r=\sqrt{p}$. At $r=\sqrt{p}, \frac{d r}{d t}=0$ but $\frac{d \theta}{d t}=1$ always. So $\theta$ is always increasing. This solution is shown in Figure C.3(b) as the solid line path that forms a circle.

Region 2: $r>\sqrt{p}$. In this region, $\frac{d r}{d t}<0$. So $r$ decreases and approaches $r=\sqrt{p}$. This solution is represented by the dotted line in Figure C.3(b).

Region 3: $r<\sqrt{p}$. In this region, $\frac{d r}{d t}>0$. Therefore $r$ increases and approaches $r=\sqrt{p}$. This solution is shown in Figure C.3(b) as the dashed line.

Consequently when $p>0, r$ approaches the constant value $\sqrt{p}$ while the angle $\theta$ constantly increases since $\frac{d \theta}{d t}=1$. Therefore, the long-term dynamics represent a point moving along a circle of radius $\sqrt{p}$. So, back in the $(x, y)$ coordinates, $x$ and $y$ are oscillating between $-\sqrt{p}$ and $\sqrt{p}$. 


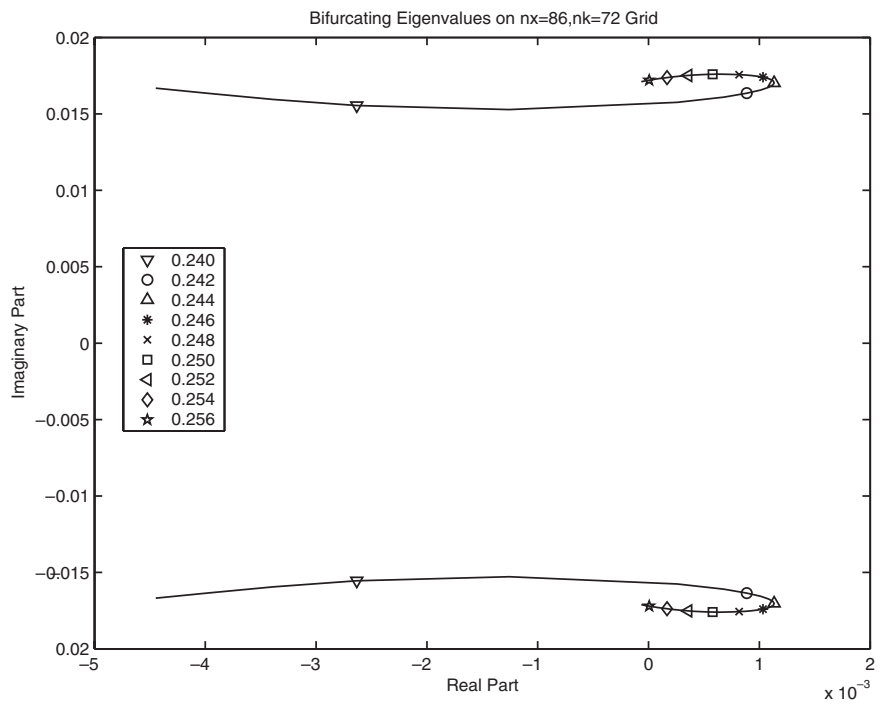

Fig. C.4 Eigenvalues of the Jacobian which create oscillatory solutions. (Legend shows voltage drop across RTD.)

To summarize, the origin is a stable equilibrium for this system when $p<0$. When $p>0$, the origin becomes unstable and an oscillatory solution to the ODE appears. This behavior can be predicted by evaluating the eigenvalues of the Jacobian of $g$, $\frac{\partial g}{\partial z}$, at the equilibrium. A Hopf bifurcation occurs if a complex-conjugate pair of eigenvalues has a real part that changes from negative to positive as the parameter is varied. In the supercritical case (illustrated above), when the real part of the eigenvalue changes sign, the equilibrium becomes unstable, and the ODE acquires a stable oscillatory solution. This is what we appear to observe below.

C.4. Back to RTDs. As illustrated in our last section, we are searching for a Hopf bifurcation in our parameter space, the applied voltage $V$. The main idea is to use continuation methods to trace the steady-state solutions $f$ for various voltages $V$. As we compute these steady-state solutions, we are inspecting the eigenvalues of the Jacobian of $W, \frac{\partial W}{\partial f}$, to find a complex-conjugate pair of eigenvalues whose real part is switching from negative to positive. Preliminary research has discovered a part of the parameter space where this may occur.

Figure C.4 presents the complex-conjugate pair of eigenvalues of $\frac{\partial W}{\partial f}$ that create this oscillatory behavior. The $x$-axis represents the real part of the eigenvalues, and the $y$-axis represents the imaginary part of the eigenvalues. The legend shows the different voltages at which these eigenvalues were calculated. At $V=0.240$ volts, the real part is negative, so we expect a stable equilibrium with no oscillation. As the voltage is increased to 0.256 volts, the real part becomes positive and stays positive. So the stability of the equilibrium is lost, and we expect oscillatory behavior. At $V=$ 0.256 volts, the real part is almost negative again. These calculations were performed with LOCA (Library of Continuation Algorithms) [27], a software library developed at Sandia National Laboratory to implement continuation algorithms. Figure C.5 is a plot of the current density versus time for various voltage differences. Current density is the current produced per area of the device. The $x$-axis represents time in 


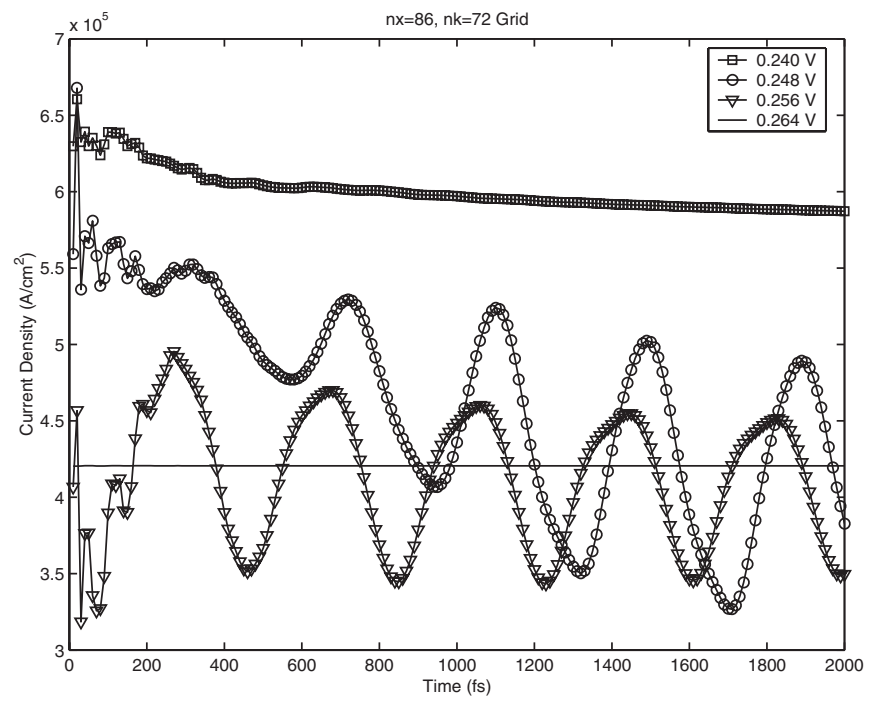

Fig. C.5 Current density versus time. (Legend shows voltage drop across RTD.)

femtoseconds $\left(10^{-15}\right.$ seconds), the $y$-axis represents current density, and the legend shows what voltage is being applied across the device. As predicted by the eigenvalue study, the current oscillates when the applied voltage is 0.248 volts and 0.256 volts, but at 0.240 volts and 0.264 volts, there is no oscillation. The current plots in Figure C.5 were produced by VODEPK $[23,24]$, a time-integration code developed at Lawrence Livermore National Laboratory.

The simulations presented in this article are numerical approximations to the Wigner-Poisson model of electron transport within nanoscale semiconductor devices. If scientists can understand how varying the applied voltage drop across the RTD leads to current oscillations, they can use the voltage drop to control the development of this oscillation. In the future, we want to establish without a doubt the existence of this Hopf bifurcation by performing more accurate computer simulations, and in particular making sure the bifurcation is not an artifact of the numerical approximations. Then this tool can be used to guide physicists and engineers in developing device structures that make effective high-frequency electronic devices.

\section{Part D. A Shocking Discovery: Nonclassical Waves in Thin Liquid Films. By Rachel Levy.}

Abstract. When a thin film flows down an inclined plane, a bulge of fluid, known as a capillary ridge, forms on the leading edge and is subject to a fingering instability in which the fluid is channeled into rivulets. This process is familiar to us in everyday experiments such as painting a wall or pouring syrup over a stack of pancakes. It is also observed that changes in surface tension due to a temperature gradient can draw fluid up an inclined plane. Amazingly, in this situation the capillary ridge broadens and no fingering instability is observed. Numerical and analytical studies of a mathematical model of this process led to the discovery that these observations are associated with a nonclassical shock wave previously unknown to exist in thin liquid films. 
Key words. thin liquid films, surface tension, classical waves, nonclassical waves

AMS subject classifications. 35K55, 76D08, 76D33, 76D 45

D.I. Introduction. Thin liquid films coat surfaces, such as computer chips, with layers as thin as thousandths of a millimeter. Ideally, the layer should be perfectly even. In reality, however, thin liquid films are uneven, leading mathematicians and scientists to study how thin films form and why they are not uniform in height.

Thin films exhibit many properties that interest mathematicians. At the leading edges of the film, fingering instabilities develop, which look like drips of paint and cause the film edge and height to be uneven [40, 41, 47, 56]. A second issue is the not yet understood mechanism for movement at the leading edge of the film, the contact line where air, liquid, and solid meet $[34,43,50,53]$. We will focus on a third issue: two kinds of shock waves that occur in the thin films experiment, and how they affect the capillary ridge, a bulge of fluid near the leading edge of the film.

In the 1990s, Anne Marie Cazabat and her colleagues in Physics at the College de France in Paris designed an experiment to investigate thin films of silicone oil coating a silicon wafer $[40,42]$. Surprising experimental results led to the discovery of nonclassical waves in thin liquid films.

D.2. The Experiment. Cazabat's thin film experiment takes place on an inclined brass plate with a reservoir of silicone oil at the bottom end (see Figure D.1). The plate is heated at the bottom and cooled at the top, with a temperature difference of $45^{\circ} \mathrm{C}$. A silicon wafer prewetted with silicone oil is attached to the plate, which can be inclined from an angle of $0^{\circ}$ (horizontal) to $90^{\circ}$ (vertical).

There are two opposing forces at work in the experiment. Since surface tension decreases with temperature, the surface tension is lower at the bottom where the film is hotter. At the top of the plate, where the film is cooler, the surface tension is higher. Consequently, a thin film of oil is pulled up the plate toward the region with higher surface tension, a phenomenon called a Marangoni force. At the same time, the oil is pulled down the plate by gravity. In the experiment, the change in temperature is large enough that the upward surface tension force exceeds the downward force of gravity, and the oil creeps up the wafer at a speed of a few centimeters per hour.

The thin films that develop on the plate are flat as they emerge from the reservoir, dip slightly before the buildup of fluid known as the capillary ridge, then slope steeply
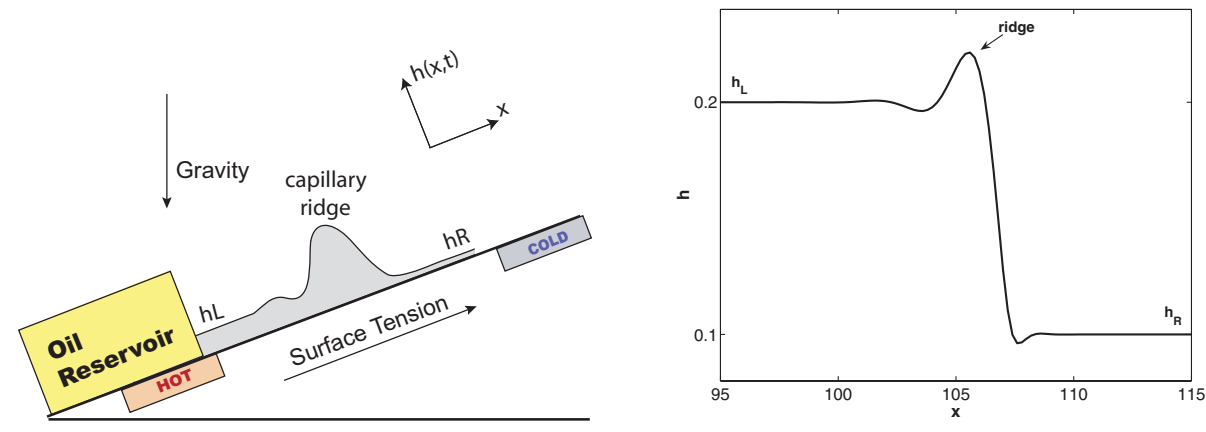

Fig. D.I On the left is a schematic of the thin film experiment viewed from the side. On the right is a numerical solution of the PDE (D.8) showing a similar profile, including the capillary ridge where the solution reaches its maximum height. 
down to the height of the prewetted surface (see Figure D.1). These features can be analyzed in one spatial dimension and time. We model the height of the film $h(x, t)$ as a function of position $(x)$ and time $(t)$ with a fourth-order nonlinear partial differential equation $(\mathrm{PDE})$

$$
h_{t}+\left(h^{2}-h^{3}\right)_{x}=-S\left(h^{3} h_{x x x}\right)_{x} .
$$

The boundary conditions for the PDE include the height emerging from the reservoir, $h=h_{L}$, and the height of the prewetting layer, $h=h_{R}$. As an initial condition, we use a smooth approximation of piecewise constant data jumping down from $h(x, 0)=h_{L}$ for $x<15$ to $h(x, 0)=h_{R}$ for $x>15$. Here $x=15$ represents the position at which the oil emerges from the reservoir.

In the PDE, the left-hand side of the equation models how the film moves and is called convective transport. In the second term, $f(h)=h^{2}-h^{3}$ represents the flux, the amount of fluid moving past a particular point at a particular time, due to the Marangoni force $\left(h^{2}\right)$ and gravity $\left(h^{3}\right)$. The right-hand side of the equation contains a fourth derivative term that represents the surface tension of the oil. This property quantifies how the oil tends to pull together (like a higher surface tension fluid such as liquid mercury that beads up) or spread out (like a low surface tension fluid such as soapy water). The variables and the equation have been derived using the wellknown lubrication approximation $[37,44]$ which takes advantage of the thinness of the film and eliminates terms that become negligible as the film thickness decreases. The equation is nondimensionalized and terms are balanced so that material properties such as viscosity and surface tension are lumped into the positive coefficient $S$ [39].

Solutions of (D.8), such as the numerical simulation in Figure D.1, agree with the expected behavior of the thin films in the experiment at large inclination angles. However, when Cazabat adjusted the inclination angle from a nearly vertical angle, such as $85^{\circ}$, to a flat angle, such as $15^{\circ}$, she observed dramatically different results. At a large inclination angle the film travels all at one speed. This means the side profile of the film, including the capillary ridge, does not change over time once the profile has formed, as in Figure D.2 (left). This is called a classical wave, which is
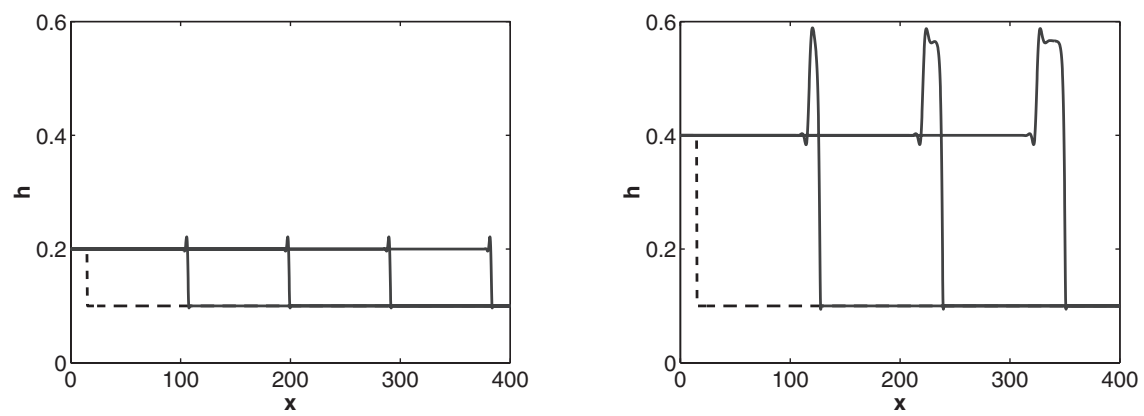

Fig. D.2 Numerical simulation of shock waves in thin liquid films. Initial condition $h(x, 0)$ is the dashed line. Film is moving to the right and is plotted at equally spaced time intervals, with the latest profile at the far right. Left plot contains a classical wave with one wave speed, corresponding to the experiment at a large inclination angle (a magnification of one profile is the right plot of Figure D.1). Right plot contains a leading faster nonclassical wave and a trailing slower classical wave, with broadening capillary ridge, corresponding to the experiment at a smaller inclination angle. 
well understood through the theory of Lax and OleĬnik which originated in the 1950s $[48,54,55]$. In contrast, at a small inclination angle the film travels at two speeds. The leading part of the film travels faster and the trailing part travels slower, causing the capillary ridge to widen over time, as in Figure D.2 (right). The mystery of the spreading capillary ridge cannot be explained by classical wave theory.

Cazabat communicated the strange results to Andrea Bertozzi at Duke University, who had previously published results on thin films [35]. Bertozzi formed a collaboration with Andreas Münch visiting Duke from Technische Universität München in Germany and my doctoral advisor, Michael Shearer, from North Carolina State University to consider the possibility of having classical and nonclassical waves in a thin liquid film $[45,46]$.

D.3. Classical and Nonclassical Waves. To understand the difference between classical and nonclassical shock waves, two graphs are helpful. In both graphs we fix a prewetting height of $h_{R}=0.1$ and vary the height $h_{L}$ at which the oil emerges from the reservoir. The first type of graph, shown in Figure D.2, represents numerical solutions of (D.8). The left plot of Figure D.2 shows a classical wave as it moves from left to right and is plotted at eight equally spaced time intervals. This wave has a short initial profile (dashed) with $h_{L}=0.2$ and $h_{R}=0.1$. The classical wave moves at a single speed and the capillary ridge maintains a constant shape, which matches the results of the experiment with a large inclination angle. The right plot of Figure D.2 contains a pair of waves: a leading faster nonclassical wave and a trailing slower classical wave, plotted at equally spaced times. This solution has a tall initial profile with $h_{L}=0.4$ and $h_{R}=0.1$. The capillary ridge widens, which agrees with experimental observations at a smaller inclination angle.

D.4. The Flux Function. The nonconvex flux function $f(h)=h^{2}-h^{3}$ is essential to the existence of nonclassical waves [37, 38]. This flux function is depicted in Figure D.3 with a black dotted curve. Each wave from Figure D.2 appears on this graph as a chord; the speed of each wave is exactly the slope of the corresponding chord.

Whether there is a single classical wave or a double wave structure depends on a critical nucleation value $h^{N}$, which in turn depends on $h_{R}$. (See Table D.1.) For

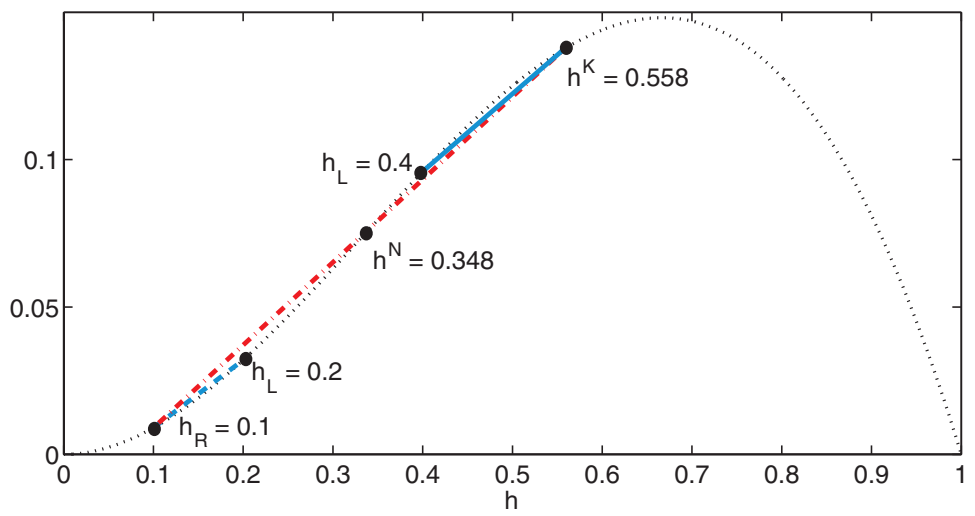

Fig. D.3 Plot of the flux function $f(h)=h^{2}-h^{3}$ (dotted black curve). The dashed blue chord from $h_{L}=0.2$ to $h_{R}=0.1$ represents the classical shock waves from Figure D.2 (left). The solid blue chord from $h_{L}=0.4$ to $h^{K}=0.558$ represents the trailing classical shock from Figure D.2 (right). The dash-dotted red chord from $h^{K}=0.558$ to $h_{L}=0.1$ represents the leading nonclassical shock wave from Figure D.2 (right). 
Table D.I Nucleation determines the types of wave structures emerging from monotonic initial data. The initial film height is piecewise constant with a single jump down from $h_{L}$ to $h_{R}$.

\begin{tabular}{|c|c|}
\hline$h_{L}<h^{N}\left(h_{R}\right)$ & Single classical wave from $h_{L}$ to $h_{R}$ \\
\hline \multirow{3}{*}{$h_{L}>h^{N}\left(h_{R}\right)$} & Double wave structure with: \\
& Classical wave from $h_{L}$ to $h^{K}$ \\
& Nonclassical wave from $h^{K}$ to $h^{R}$ \\
\hline
\end{tabular}

$h_{R}=0.1, h^{N}=0.348$. If $h_{L}$ is below $h^{N}$, the film develops a single classical wave from $h_{L}$ to $h_{R}$. If $h_{L}$ is greater than $h^{N}$, the film nucleates a double wave structure $[49,51]$, consisting of a trailing slower classical wave and a leading faster nonclassical wave. The double wave structure gives rise to a second critical value, $h^{K}=0.558$, as an endpoint for each of the wave chords. The two waves in the double wave structure are, then, a classical wave from $h_{L}$ to $h^{K}$ and a nonclassical wave from $h^{K}$ to $h_{R}$.

A classical wave is one for which the corresponding chord does not pass through the dotted black curve $f(h)$. This is true for the classical waves from $h_{L}=0.2$ to $h_{R}$ represented by a dashed blue chord and from $h_{L}=0.4$ to $h^{K}$ represented by a solid blue chord. The nonclassical wave from $h^{K}$ to $h_{L}=0.4$ is represented by a dash-dotted red chord passing through the flux curve. Since the dash-dotted (red) chord has a steeper slope than the solid (blue) chord, the leading nonclassical wave is faster than the trailing classical wave and the capillary ridge between them separates. [48]

In contrast to nonclassical waves, classical waves satisfy the Lax shock inequalities

$$
f^{\prime}\left(h_{L}\right)>\frac{f\left(h_{R}\right)-f\left(h_{L}\right)}{h_{R}-h_{L}}>f^{\prime}\left(h_{R}\right) .
$$

Here the left and right terms represent the slope of the tangent to the flux function at the ends of the chord from $h_{L}$ to $h_{R}$. The center quantity is the speed of the wave, equal to the slope of the chord. The inequality implies that compared to the wave speed, the characteristic speed ahead of the wave is slower and behind the wave is faster, so that characteristics enter the wave from both sides. In Figure D.3, this requirement is satisfied by the two blue (classical) chords but not by the red (nonclassical) one for which both characteristics speeds are slower than the wave speed and the characteristics pass through the wave from front to back:

$$
\max \left(f^{\prime}\left(h_{R}\right), f^{\prime}\left(h^{K}\right)\right)<\frac{f\left(h^{K}\right)-f\left(h_{R}\right)}{h^{K}-h_{R}} .
$$

D.5. Connection between Theory and Experiment. Now we know that for a given prewetting height $h_{R}$, if the height $h_{L}$ of the emerging oil is small enough, a single classical wave develops. If $h_{L}$ is large enough, a classical/nonclassical wave pair develops instead. We can connect this information to the mysterious results of the experiment. The key is that a large inclination angle causes the oil to emerge from the reservoir at a lower height and thus form a classical wave. A small inclination angle causes the oil to emerge from the reservoir at a larger height and thus form a classical/nonclassical pair. The existence of nonclassical waves also explains another curious result in the experiment, a lack of fingering at the edge of the film in the lowangle case because the nonclassical wave stabilizes the leading edge [36, 40, 41, 56].

Bertozzi, Münch, and Shearer performed numerical experiments to explore the possibility of nonclassical waves in thin films and employed mathematical analysis to 
prove the existence of the waves in the second half of 1998 [37, 38]. This led to an NSF-funded focused research group on thin films which still meets regularly today [57]. One area of research I have pursued with Michael Shearer is to predict the types of waves that emerge for a given initial profile [49]. In 2003, we completed a map of the Riemann problem [51], which means that for (D.8) and the experiment, we can predict which types of waves emerge from monotonic initial data (piecewise constant initial profiles with a single jump up or jump down from $h_{L}$ to $h_{R}$ ).

The successful international collaboration between Cazabat's group in Europe and Bertozzi's group in the United States brought physicists and mathematicians together to explain the mysterious experimental results and paved the way to a deeper understanding of the role of classical and nonclassical waves in thin liquid films.

Acknowledgments. We thank the two referees as well as the Section Editor, Andy Bernoff, for their great care in reviewing this paper and for offering us many helpful suggestions. ML would like to thank Tim Kelley, Andrew Salinger, Dwight Woolard, and Peiji Zhao for their help and supervision. RL would like to thank Michael Shearer, Andrea Bertozzi, Tom Witelski, Mette Olufsen, and Alina Chertock for their support and encouragement.

\section{REFERENCES}

[1] N. J. Higham, Handbook of Writing for the Mathematical Sciences, 2nd ed., SIAM, Philadelphia, 1998.

[2] S. G. Krantz, A Primer of Mathematical Writing, AMS, Providence, RI, 1997.

[3] B. N. PARlett, The Symmetric Eigenvalue Problem, Prentice-Hall, Englewood Cliffs, NJ, 1980.

[4] V. RACOČEvić, On the norm of idempotent operators in a Hilbert space, Amer. Math. Monthly, 107 (2000), pp. $748-50$.

[5] D. E. STEWART, Rigid-body dynamics with friction and impact, SIAM Rev., 42 (2000), pp. 3-39.

[6] L. N. Trefethen, Introduction to "Rigid body dynamics with friction and impact," SIAM Rev., 42 (2000), p. 1.

[7] J. H. Wilkinson, The perfidious polynomial, in Studies in Numerical Analysis, G. H. Golub, ed., MAA Stud. Math. 24, Math. Assoc. America, Washington, D.C., 1984, pp. 1-28.

\section{PART A}

[8] T. D. Choi, O. J. Eslinger, P. Gilmore, A. Patrick, C. T. Kelley, and J. M. Gablonsky, IFFCO: Implicit Filtering for Constrained Optimization, Version 2, Tech. Report CRSCTR99-23, CRSC, 1999.

[9] P. A. Domenico And F. W. Schwartz, Physical and Chemical Hydrogeology, 2nd ed., John Wiley and Sons, New York, 1998.

[10] K. R. Fowler, J. P. Reese, C. E. Kees, J. E. Dennis, C. T. Kelley, C. T. Miller, C. Audet, A. J. Booker, G. Couture, R. W. Darwin, M. W. Farthing, D. E. Finkel, J. M. Gablonsky, G. Gray, and T. G. Kolda, A comparison of optimization methods for problems involving flow and transport phenomena in saturated subsurface systems, in preparation.

[11] R. Freeze and J. Cherry, Groundwater, Prentice-Hall, Englewood Cliffs, NJ, 1979.

[12] A. Harbaugh and M. McDonald, User's Documentation for MODFLOW-96: An Update to the U.S. Geological Survey Modular Finite-Difference Ground-Water Flow Model, OpenFile Report OFR 96-485, U.S. Geological Survey, 1996.

[13] C. T. Kelley, Iterative Methods for Optimization, Frontiers Appl. Math. 18, SIAM, Philadelphia, 1999.

[14] A. S. Mayer, C. T. Kelley, And C. T. Miller, Optimal design for problems involving flow and transport phenomena in saturated subsurface systems, Adv. Water Resources, 25 (2002), pp. 1233-1256.

[15] A. S. Mayer, C. T. Kelley, And C. T. Miller, Electronic supplement to "Optimal design for problems involving flow and transport phenomena in saturated subsurface systems," 2003, http://www4.ncsu.edu/ ctk/PAPERS/Mayer-Sl-v2.pdf. 


\section{PART B}

[16] S. A. Ahmed, R. Buckingham, P. A. Gremaud, C. D. Hauck, C. M. Kuster, M. ProDANovic, T. A. Royal, And V. Silantyev, Volume determination for bulk materials in bunkers, Internat. J. Numer. Methods Engrg., 61 (2004), pp. 2239-2249.

[17] G. Aronsson, A mathematical model in sand mechanics: Presentation and analysis, SIAM J. Appl. Math., 22 (1972), pp. 437-458.

[18] M. Born And E. Wolf, Principles of Optics: Electromagnetic Theory of Propagation, Interference and Diffraction of Light, Pergamon Press, New York, 1959.

[19] M. G. Crandall and P. L. Lions, Viscosity Solutions of Hamilton-Jacobi Equations, Trans. Amer. Math. Soc., 277 (1983), pp. 1-42.

[20] J. A. Sethian, Fast marching methods, SIAM Rev., 41 (1999), pp. 199-235.

[21] J. N. TsitsikLIS, Efficient algorithms for globally optimal trajectories, IEEE Trans. Automat. Control, 40 (1995), pp. 1528-1538.

\section{PART C}

[22] P. J. Antsaklis And A. N. Michel, Linear Systems, McGraw-Hill Electrical and Computer Engineering Series, McGraw-Hill, New York, 1997.

[23] P. N. Brown, G. D. Byrne, and A. C. Hindmarsh, VODE: A Variable-Coefficient ODE Solver, Technical Report UCRL-98412, Lawrence Livermore National Laboratory, Livermore, CA, 1988.

[24] P. N. Brown And A. C. Hindmarsh, Reduced Storage Matrix Methods in Stiff ODE Problems, Technical Report UCRL-95088, Lawrence Livermore National Laboratory, Livermore, CA, 1987.

[25] T. R. Globus, D. L. Woolard, T. Khromova, T. W. Crowe, M. Bykhovskaia, B. L. Gelmont, J. Helser, and A. C. Samuels, THz-spectroscopy of biological molecules, J. Biol. Phys., 29 (2003), pp. 89-100.

[26] Y. A. Kuznetsov, Elements of Applied Bifurcation Theory, 2nd ed., Appl. Math. Sci. 112, Springer-Verlag, New York, 1998.

[27] A. G. Salinger, N. M. Bou-Rabee, R. P. Pawlowski, E. D. Wilkes, E. A. Burroughs, R. B. LehoucQ, And L. A. Romero, LOCA 1.0 Library of Continuation Algorithms: Theory and Implementation Manual, Technical Report SAND2002-0396, Sandia National Laboratory, Albuquerque, NM, 2002.

[28] T. C. L. G. Sollner, W. D. Goodhue, P. E. Tannenwald, C. D. Parker, and D. D. Peck, Resonant tunneling through quantum wells at frequencies up to $2.5 \mathrm{THz}$, Appl. Phys. Lett., 43 (1983), pp. 588-590.

[29] T. C. L. G. Sollner, P. E. Tannenwald, D. D. Peck, and W. D. Goodhue, Quantum well oscillators, Appl. Phys. Lett., 45 (1984), pp. 1319-1321.

[30] E. Wigner, On the quantum correction for thermodynamic equilibrium, Phys. Rev., 40 (1932), pp. 749-759.

[31] P. Zhao, H. L. Cui, And D. L. Woolard, Dynamical instabilities and I- $V$ characteristics in resonant tunneling through double barrier quantum well systems, Phys. Rev. B, 63 (2001), article 75302 .

[32] P. Zhao, D. L. Woolard, B. L. Gelmont, and H. L. Cui, Creation and quenching of interference-induced emitter-quantum wells within double-barrier tunneling structures, J. Appl. Phys., 94 (2003), pp. 1833-1849.

\section{PART D}

[33] D. J. Acheson, Elementary Fluid Dynamics, Oxford University Press, Oxford, UK, 1990.

[34] A. L. BertozzI, The mathematics of moving contact lines in thin liquid films, Notices Amer. Math. Soc., 45 (1998), pp. 689-697.

[35] A. L. Bertozzi And M. P. Brenner, Linear stability and transient growth in driven contact lines, Phys. Fluids, 9 (1997), pp. 530-539.

[36] A. L. Bertozzi, A. Münch, M. Shearer, and K. Zumbrun, Stability of compressive and undercompressive thin film travelling waves, European J. Appl. Math., 12 (2001), pp. 253291.

[37] A. L. Bertozzi, A. Münch, and M. Shearer, Undercompressive shocks in thin film flow, Phys. D, 134 (1999), pp. 431-464.

[38] A. L. Bertozzi and M. Shearer, Existence of undercompressive traveling waves in thin film equations, SIAM J. Math. Anal, 32 (2000), pp. 194-213.

[39] R. Buckingham, M. Shearer, and A. Bertozzi, Thin film traveling waves and the Navier slip condition, SIAM J. Appl. Math., 63 (2003), pp. 722-744. 
[40] A. M. Cazabat, F. Heslot, S. M. Troian, and P. Carles, Fingering instability of thin spreading films driven by surface tension gradients, Nature, 346 (1990), pp. 824-826.

[41] E. B. V. Dussan AND S. Davis, On the motion of a fluid-fluid interface along a solid surface, J. Fluid Mech., 65 (1974), pp. 71-95.

[42] X. Fanton, A. M. Cazabat, And D. QuÉRÉ, Thickness and shape of films driven by a Marangoni flow, Langmuir, 12 (1996), pp. 5875-5880.

[43] P. G. DE Gennes, Wetting: Statics and dynamics, Rev. Modern Phys., 57 (1985), pp. 827-863.

[44] H. P. Greenspan, On the motion of a small viscous droplet that wets a surface, J. Fluid Mech., 84 (1978), pp. 125-143.

[45] B. Hayes And M. Shearer, Undercompressive shocks and Riemann problems for scalar conservation laws with non-convex fluxes, Proc. Roy. Soc. Edinburgh Sect. A, 129 (1999), pp. $733-775$.

[46] D. Jacobs, W. McKinney, And M. Shearer, Travelling wave solutions of the modified Korteweg-deVries equation, J. Differential Equations, 116 (1995), pp. 448-467.

[47] L. Kondic, Instabilities in gravity driven flow of thin fluid films, SIAM Rev., 45 (2003), pp. 95-115.

[48] P. D. LAx, Hyperbolic Systems of Conservation Laws and the Mathematical Theory of Shock Waves, CBMS-NSF Regional Conf. Ser. in Appl. Math. 11, SIAM, Philadelphia, 1973.

[49] P. G. LeFloch and M. Shearer, Nonclassical Riemann solvers with nucleation, Proc. Roy. Soc. Edinburgh Sect. A, 134 (2004), pp. 961-984.

[50] R. Levy AND M. Shearer, Comparison of dynamic contact line models for driven thin liquid films, European J. Appl. Math., 15 (2004), pp. 625-642.

[51] R. Levy And M. Shearer, Kinetics and nucleation for driven thin film flow, Phys. D, 209 (2005), pp. 145-163.

[52] C. C. Lin and L. A. Segel, Mathematics Applied to Deterministic Problems in the Natural Sciences, Classics Appl. Math. 1, SIAM, Philadelphia, 1988.

[53] V. Ludviksson And E. N. Lightfoot, The dynamics of thin films in the context of surface tension gradients, AIChE J., 17 (1971), pp. 1166-1173.

[54] O. A. OLĚ̆NIK, Uniqueness and stability of the generalized solution of the Cauchy problem for a quasi-linear equation, Uspekhi Mat. Nauk., 14 (1959), pp. 165-170.

[55] O. A. OlEINIK, Discontinuous solutions of nonlinear differential equations, Amer. Math. Soc. Transl., 26 (1963), pp. 95-172.

[56] J. Sur, A. L. Bertozzi, And R. P. Behringer, Reverse undercompressive shock structures in driven thin film flow, Phys. Rev. Lett., 90 (2003), article 126105.

[57] Website for focused research group in thin films and fluid interfaces organized by Behringer (Duke), Bertozzi (UCLA), Shearer (NCSU), and Witelski (Duke): http://www.math.duke. edu/ witelski/thinfilms/. 